\title{
Crime sans châtiment
}

\author{
F. Klotz \\ C) Springer-Verlag France 2010
}

L'homme est capable du meilleur comme du pire, et le pire n'a pas de limites dans la négation de toutes les valeurs morales.

L'inondation du monde par les faux médicaments est une réalité de notre siècle. Les fabricants sans aucun scrupule forment un nouveau cartel qui n'a rien à envier à celui de la drogue.

Le marché est estimé entre 25 et 40 milliards d'euros par an et représenterait $6 \%$ du marché mondial de l'industrie pharmaceutique, avec des variations considérables selon les pays : de $60 \%$ dans les pays les plus pauvres d'Afrique, où les médicaments sont vendus sur les marchés au milieu du bazar hétéroclite des marchandises, à moins de $1 \%$ en Europe de l'Ouest, en passant par $20 \%$ en Russie et $40 \%$ dans les pays défavorisés d'Amérique latine.

Les contrefaçons de produits pharmaceutiques ont explosé sur Internet, avec la vente de faux viagra ou de faux antidépresseurs, lésant les occidentaux crédules. Ce qui est beaucoup plus grave, c'est la production industrielle et la vente de faux antipaludéens, de faux antibiotiques, de faux antidiabétiques, de faux antihypertenseurs, de faux antiulcéreux à des prix attractifs dans des officines illégales et sur les marchés des pays pauvres d'Afrique noire et d'Asie du Sud-Est en particulier.

On meurt aujourd'hui dans ces pays de paludisme et de pneumonie, victime de faux médicaments dépourvus de principe actif. C'est insoutenable !

Ces produits factices ou insuffisamment dosés viennent de Chine, d'Inde, du Nigeria ou d'ailleurs, où des fabricants criminels et sans scrupules font des bénéfices allant parfois à plus de $10000 \%$, bien supérieurs à ceux du trafic des drogues dures.

Il faut se rappeler que l'achat de médicaments dans les pays pauvres dépourvus de couverture sociale représente le deuxième poste dans le budget des familles qui sont naturellement tentées de chercher le produit le moins cher, souvent contrefait.

Quand on sait qu'en Asie du Sud-Est $40 \%$ des antipaludéens, supposés à base d'artésunate, ne contiennent aucun principe actif ; quand on rapporte que du talc est vendu à la place de l'aspirine sur les marchés du Burkina Faso, que des collyres contenant de l'eau croupie, que des pommades contenant de la sciure sont sur la place publique au Nigeria ; quand on apprend que le sirop de paracétamol pour enfant a été remplacé par de l'antigel et qu'il a causé la mort de 89 personnes en Haïti en 1995 et celle de 30 nourrissons en Inde en 1998, on est atterré sur la nature humaine !

Ces faits émergents ne sont que la petite pointe de l'iceberg de ce trafic.

La conférence de Cotonou qui vient de se tenir sur ce thème, sous l'égide de la fondation Chirac et en présence de plusieurs chefs d'État africains, a permis à nouveau d'éveiller les consciences sur ce crime organisé qui pourrait être responsable de la mort de plus de 200000 personnes par an dans la ceinture de la pauvreté !

Si prendre un faux comprimé de viagra n'a pas de conséquence vitale, traiter un paludisme de l'enfant de toute bonne foi avec des faux comprimés d'artésunate peut être gravissime.

Les gastroentérologues du continent africains doivent être conscients de la supercherie criminelle qui se déroule chez eux pour le profit de gens sans scrupules et au détriment des malades. L'achat des médicaments doit impérativement se faire dans les pharmacies officielles garantissant la qualité des produits vendus et leur origine. Cela doit être expliqué à chaque malade lors de la consultation. Mais il faut aussi que les pouvoirs publics traquent ces malfaiteurs de manière active et leur inflige de lourdes peines, car de tels crimes doivent avoir un châtiment exemplaire.

F. Klotz $(\bowtie)$

e-mail : fklotz2008@yahoo.fr 